\title{
Application of Soft X-Ray Imaging System to the STE-2 RFP
}

\author{
Takumi ONCHI, Akio SANPEI, Ryuya IKEZOE, Haruhiko HIMURA and Sadao MASAMUNE \\ Kyoto Institute of Technology, Kyoto 606-8585, Japan \\ (Received 9 December 2006 / Accepted 26 April 2007)
}

\begin{abstract}
A soft X-ray (SXR) imaging system (consisting of a microchannel plate, a phosphor plate and an image intensifier CCD camera) has been installed in the STE-2 RFP $(R / a=0.4 \mathrm{~m} / 0.1 \mathrm{~m})$ to obtain high-resolution twodimensional (2-D) SXR images to see if 3-D magnetic structures can be deduced from the 2-D SXR image. The SXR images obtained in standard RFP plasmas have been compared with those in RFP plasmas with different MHD properties. We have identified characteristic structures in the 2-D SXR images depending upon the MHD properties with the present system. Optimization of the image data processing such as noise filtering from the data or tone correction of brightness distribution is also discussed.
\end{abstract}

(C) 2007 The Japan Society of Plasma Science and Nuclear Fusion Research

Keywords: soft X-ray, imaging, RFP, magnetic island, pinhole camera

DOI: $10.1585 /$ pfr.2.S1063

\section{Introduction}

The reversed field pinch (RFP) is one of the toroidal magnetic confinement systems for compact, high-beta plasmas for nuclear fusion reactor [1]. The RFP configuration is formed and sustained as a result of nonlinear MHD phenomena such as MHD relaxation and RFP dynamo. Recent progress in the RFP research has revealed the importance of resistive tearing modes in the nonlinear MHD dynamics [2]. In particular, active control of the MHD dynamics is essential to the confinement improvement [3].

In typical RFP configuration, the safety factor decreases towards the edge, with on-axis value $q_{0}$, of about $0.5-0.7 \times(a / R$ (inverse aspect ratio) $)$ and negative value outside the field reversal surface. The mode rational surfaces of the $m=1$ tearing modes are therefore rather closely spaced over the minor cross section. The toroidal mode numbers of the dominant modes lie around the aspect ratio, and therefore, observation of magnetic island structures with short toroidal pitch lengths is quite important in the study of MHD dynamics and their active control [4].

We have been developing a simple soft X-ray (SXR) imaging system which has the capability of providing 3$\mathrm{D}$ magnetic island structure [5]. The system has been installed in the STE-2 [6,7] RFP to carry out the proof-ofprinciple experiment by observing SXR images of RFP plasmas with different MHD characteristics. In the present experiment, resonant rotating helical field (RHF) has been applied to control the MHD dynamics, and we have observed the influence of change in MHD characteristics in the SXR image.

The STE-2 is a small RFP with aspect ratio of $4(R / a=0.4 \mathrm{~m} / 0.1 \mathrm{~m})$. In typical RFP configuration, the safety factor is about 0.15 on axis, decreasing towards the edge to about -0.05 . Thus the dominant toroidal mode

author'se-mail: onchi5t@nuclear.dj.kit.ac.jp numbers $n$ of the $m=1$ magnetic fluctuation are in the range from 7-10. The SXR images were obtained in these standard RFP plasmas. We have made use of the rotating helical field to change the MHD characteristics to observe SXR images.

We have identified characteristic structures in the 2-D SXR images obtained with the present system, which may be an indication of the helical structure in the RFP plasma. The observed structure appeared to show dependence on the RFP discharge conditions. Optimization of the image data processing such as noise filtering from the data or tone correction of brightness distribution is in progress to realize effective visualization of the deduced 3-D magnetic structure. Detailed analysis of the structures in the SXR images has also been carried out together with analysis of magnetic fluctuation data.

\section{Experimental Arrangement}

The SXR imaging system consists of the pinhole, MCP, phosphor plate and ICCD camera. The minor cross section of the experimental arrangement is shown in Fig. 1. The pinhole of $1 \mathrm{~mm}$ diameter is placed at $35 \mathrm{~mm}$ away from the plasma surface in the major radial direction on the equatorial plane. Since the MCP (Hamamatsu Photonics F2222-21P) is sensitive to SXR but insensitive to visible light, SXR images are obtained without any filter. In addition, SXR images filtered by two kinds of polyester foils of $1.5 \mu \mathrm{m}$ and $3.0 \mu \mathrm{m}$ thickness have been obtained to realize a rough energy analysis. The entrance surface of MCP is $50 \mathrm{~mm}$ away from the pinhole. The optical system from the phosphor plate to ICCD is designed so that the phosphor image is formed as a circle of 150-pixel diameter on the ICCD plate.

A 2-D distribution of secondary electrons induced by the X-ray image is amplified through a bundle of capil- 


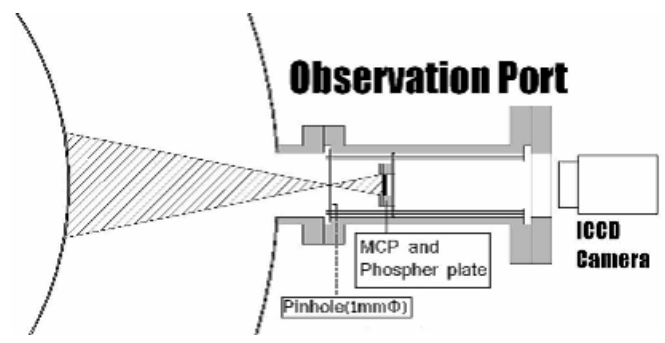

Fig. 1 Experimental arrangement.

laries with $20 \mathrm{~mm}$ diameter by an externally applied bias voltage $-1.0 \mathrm{kV}$. The electrons reaching the end surface of the MCP are accelerated by $2.0 \mathrm{kV}$, which is applied to the surface of the phosphor screen, toward a phosphor screen to generate a visible light image of the secondary electron distribution. This process is completed in a vacuum chamber.

The 2-D luminosity distributions on the phosphor plate are measured with a high-speed ICCD camera with $385 \times 579$ pixels array and 32 bits of dynamic range. In the present study, SXR image is produced in $150 \times 150$ pixels area on ICCD. The dynamic range of the ICCD camera's sensitivity is controlled with the exposure time. In most cases, we set the exposure time to $5 \mu \mathrm{sec}$ at the required time of observation.

Space limitations by observation port and system arrangement for the principle verification allow of only narrow angle horizontal view $\left(24^{\circ}\right)$. To test the sensitivity of the SXR images due to region of the magnetic island in field of view to the change in parameter for discharge is major aim of this experiment, rather than to observe the magnetic island structure itself directly.

\section{Results and Discussion}

The SXR imaging system has been installed in the STE-2 RFP to obtain SXR images under different discharge conditions. The STE-2 is a small RFP $(R=0.4 \mathrm{~m} / a=0.1 \mathrm{~m})$ with aspect ratio of 4 . The major purpose of the present experiment is to see if we can identify any structure on the 2-D SXR image depending upon the RFP plasma characteristics. Although the viewing area is restricted to about $10 \mathrm{~cm}$ in diameter on axis due to the size of diagnostic port available, the present experiment corresponds to the proof-of-principle of the developed SXR imaging system.

Figure 2 shows time evolution of the plasma current $I_{p}$, toroidal magnetic field at the edge $B_{z}(a)$ and average toroidal field $\left\langle B_{z}\right\rangle$ of a typical RFP plasma in STE-2. The maximum plasma current is about $40 \mathrm{kA}$ with discharge duration of about $0.6 \mathrm{~ms}$. The SXR image is taken at the current maximum, $0.5 \mathrm{~ms}$ into the discharge.

In the present experimental arrangement, the diameter of resonant surface for the dominant $m=1 / n=8$ mode is larger than the viewing area. Therefore, a direct image of

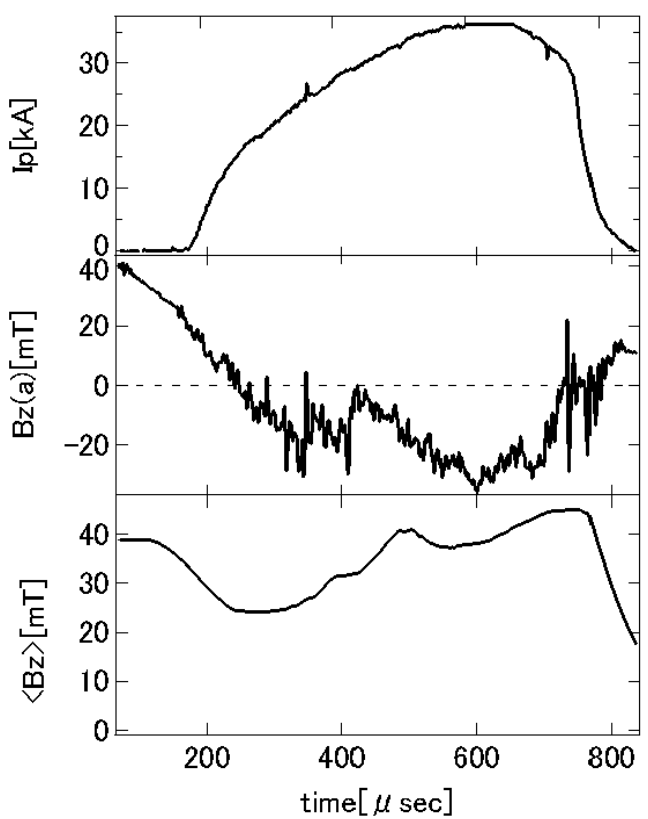

Fig. 2 Time evolution of RFP parameters.

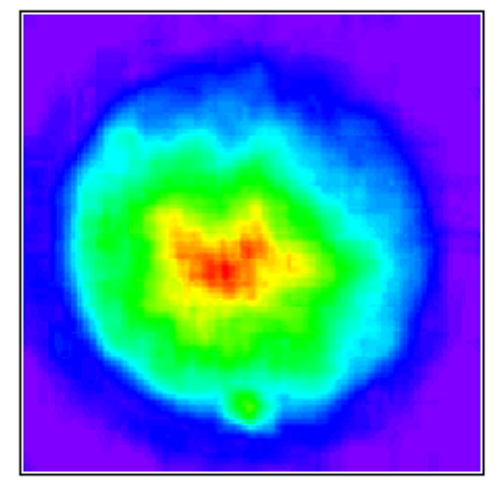

Fig. 3 A SXR image from standard RFP.

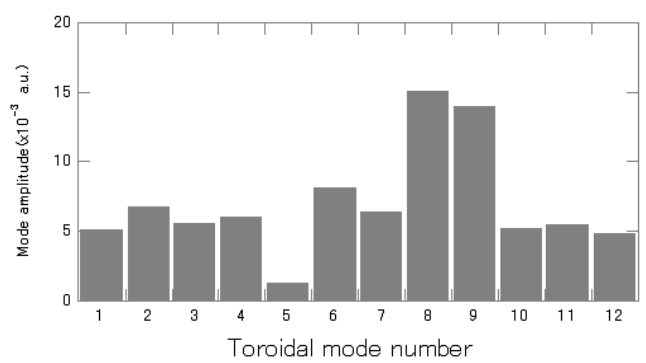

Fig. 44 Toroidal mode spectrum of $m=1$ magnetic fluctuations in standard RFP.

the island is invisible when it is located either upper or lower edge in the poloidal cross section. Nevertheless, we can identify the characteristics of the magnetic island in the SXR image depending on the phase of the island, because it contains information on the luminosity distribution integrated along the line-of-sight. 


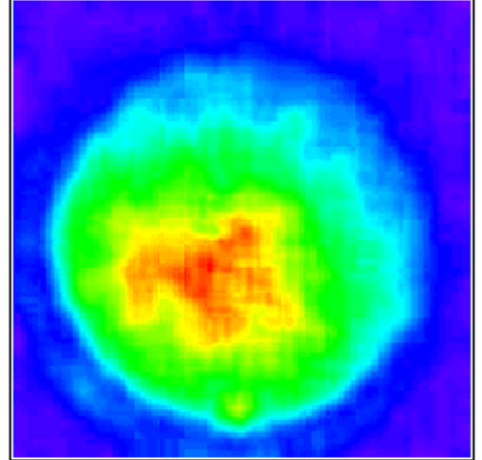

Fig. 5 A SXR image from RFP with MHD characteristic different from in Fig. 3.

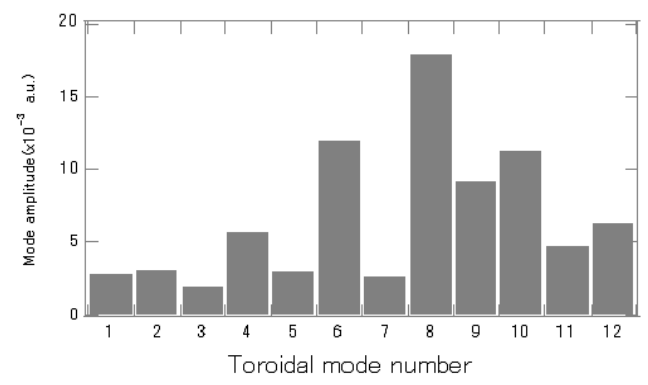

Fig. 6 Toroidal mode spectrum different from standard RFP case.

Figure 3 gives an example of the SXR image in a typical RFP plasma. We can identify a bright blob or two blobs near the left hand side to the center in the image. Figure 4 shows the toroidal mode spectrum of the $m=1$ magnetic fluctuations time averaged over $50 \mu$ s before the start of ICCD gate for the purpose of comparison of the SXR image with MHD mode activity. The duration of time average of $50 \mu \mathrm{s}$ corresponds to the duration of residual light of phosphor plate, because the SXR image is a time-integrated image due to the residual light of phosphor plate. The dominant modes are $m=1 / n=8,9$. The mode spectrum of edge magnetic fluctuation in Fig. 4 shows that $m=1 / n=8,9$ modes have higher amplitude than the other modes.

Figure 5 gives the SXR image in an RFP with characteristic different from that in standard one. Bright blob is not so clear in this image when compared with that in Fig. 3. We may be able to identify an elliptic region with an inclination angle opposite to that in Fig. 3. The $M / N=1 / 8$ resonant rotating helical field (RHF) with $10 \mathrm{kHz}$ has been applied from outside of vessel to change the MHD mode behavior, where $M(N)$ is the poloidal (toroidal) mode number of the external rotating field. Figure 6 gives the toroidal mode spectrum of the $m=1$ mode in the case of Fig. 5 . The spectrum is obtained by the same procedure as in Fig. 4. The $m=1 / n=8$ mode is dominant and $m=1 / n=6$ mode has a significant amplitude in this case. A simple equilib-

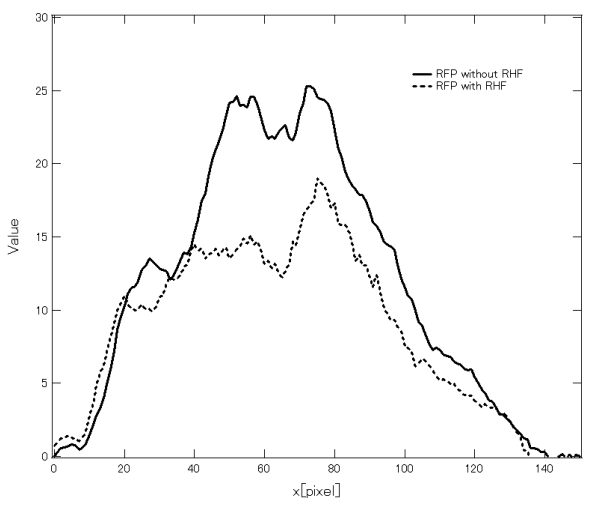

Fig. 7 Intensity distributions along the horizontal line in the SXR images in Figs. 3 and 5.

rium model suggests that the $m=1 / n=6$ mode is nonresonant in the present case, so, the $m=1 / n=6$ may correspond to a global deformation of the plasma column. Typical $q$ profile in STE-2 RFP plasma shows that $q_{0}<1 / 6$, indicating that $m=1 / n=6$ mode is internally non-resonant. The non-resonant mode causes global deformation of the plasma column, resulting in enhanced plasma-wall interaction with lowering the electron density. Thus, when the amplitude of $m=1 / n=6$ mode is high, we expect lower SXR intensity mainly because of the lower temperature.

Figure 7 compares the intensity distribution along the horizontal line of the images in Figs. 3 and 5. In both cases, we can identify a sort of local structure (peak or peaks) in the distribution, and the structure changes with the change in RFP plasma characteristics. In the case of standard RFP plasma, the mode spectrum shows two dominant resonant modes. If magnetic island is formed for each mode on its resonant surface, we may expect constructive contribution from these islands to the SXR image, depending upon the phase relation of these modes. On the other hand, in the case of RFP with MHD characteristics shown in Fig. 5, the dominant mode is $n=8$ with the same amplitude as in standard RFP. Taking into account the global deformation of $m / n=1 / 6$, we may expect lower intensity of SXR image, also depending upon the phase relation of these modes. Further detailed analyses of the $m=1$ modes is in progress to make clear the phase relation among various $m=1$ modes at the toroidal location of SXR image observation.

\section{Conclusion}

We have developed an SXR imaging system for obtaining 3-D information on magnetic structures in RFP plasmas. We have succeeded in obtaining SXR images, and identified a sort of change in local structure of the image, depending upon the RFP plasma characteristics. Further analysis techniques such as numerical modeling may be required for the detailed discussion on magnetic structures. 
[1] D.C. Robinson, Nucl. Fusion 18, 939 (1978).

[2] N. Mattor et al., Phys. Plasmas 3, 1578 (1996).

[3] R. Paccagnella, D. Gregoratto and A. Bondeson, Nucl. Fusion 42, 1102 (2002).

[4] H.K. Moffat, Magnetic Field Generation in Electrically Conducting Fluids (Cambridge University Press, Cambridge, 1978).
[5] A. Sanpei, T. Onchi, R. Ikezoe et al., poster P6-27 in this conference (2006).

[6] S. Masamune et al., Proc. 18th IAEA Fusion Energy Conf., IAEA/F1-CN-77/EXP3/11 (2001).

[7] S. Masamune and M. Iida, J. Plasma Fusion Res. Series, 5, 509 (2003). 